\title{
CpG Oligodeoxynucleotides Attenuate OVA-Induced Allergic Airway Inflammation via Suppressing JNK-Mediated Endoplasmic Reticulum Stress
}

\author{
Hai-Yun Zhang ${ }^{1-3}$ \\ Qiu-Meng Xie ${ }^{1-3}$ \\ Cui-Cui Zhao ${ }^{1-3}$ \\ Jia-Feng Sha ${ }^{1-3}$ \\ Ya Ruan ${ }^{1-3}$ \\ Hui-Mei Wu (D) ${ }^{1-3}$
}

'Anhui Geriatric Institute, Department of Geriatric Respiratory and Critical Care, The First Affiliated Hospital of Anhui Medical University, Hefei, Anhui, People's Republic of China; ${ }^{2}$ Key Laboratory of Geriatric Molecular Medicine of Anhui Province, Hefei, Anhui, People's Republic of China; ${ }^{3}$ Key Laboratory of Respiratory Disease Research and Medical

Transformation of Anhui Province, Hefei, Anhui, People's Republic of China
Correspondence: Hui-Mei Wu

Anhui Geriatric Institute, Department of Geriatric Respiratory and Critical Care,

The First Affiliated Hospital of Anhui

Medical University, Hefei, Anhui, People's

Republic of China

Tel +8655I 65I20742

Email wuhm@ahmu.edu.cn
Purpose: CpG-ODN has been found to attenuate allergic airway inflammation in our previous study. Here, we aimed to further investigate whether CpG-ODN exerts such effect via regulating endoplasmic reticulum (ER) stress and revealed the underlying mechanism.

Methods: Five-week-old C57BL/6 mice were randomly grouped and treated with or without CpG-ODN or/and SP600125. Meantime, RAW264.7 cells were used to investigate the effect of CpG-ODN on OVA-induced ER stress in vitro. The cellularity of bronchoalveolar lavage fluid (BALF) was classified and counted after Wright-Giemsa staining. HE and PAS staining methods were applied to analyze airway inflammation. The protein levels of IL-4, IL-5, IL13, p-JNK, JNK, CHOP, XBP1, ATF6 $\alpha$ and GRP78 in lung tissues were detected by Western blotting. Correspondingly, the ER stress markers were detected by Western blotting and immunofluorescence in RAW264.7 cells.

Results: In OVA-induced allergic airway inflammation, CpG-ODN significantly suppressed inflammatory cells infiltration, goblet cell hyperplasia and the protein expression of $\mathrm{Th} 2$ cytokines. Moreover, OVA exposure strongly increased the activation of ER stress with higher protein expressions of CHOP, XBP1, ATF6 $\alpha$ and GRP78. However, these OVAinduced increase of ER stress markers were markedly suppressed by CpG-ODN treatment. In addition, exposure to OVA significantly increased the phosphorylation of JNK, which was significantly reduced by CpG-ODN treatment. Remarkably, single treatment of SP600125, an antagonist of JNK, functioned similarly as CpG-ODN in mitigating allergic airway inflammation and suppressing OVA-induced activation of ER stress; however, no significant synergistic effect was evidenced by combined treatment of SP600125 and CpG-ODN. Furthermore, in OVA-stimulated RAW264.7 cells, we also found that OVA stimulation increased the expressions of ER stress markers, and CpG-ODN significantly reduced their expression levels via suppressing the phosphorylation of JNK.

Conclusion: These results indicated that $\mathrm{CpG}-\mathrm{ODN}$ mitigates allergic airway inflammation via suppressing the activation of JNK-medicated ER stress.

Keywords: asthma, CpG-ODN, SP600125, endoplasmic reticulum homeostasis

\section{Plain Language Summary}

Improvements in allergic airway inflammation may be achieved by treatments that suppress the allergen-induced endoplasmic reticulum stress in the lung. Our study shows that immune adjuvant CpG-ODN that suppresses the JNK-medicated endoplasmic reticulum stress effectively mitigates the development of allergic airway inflammation pathology in vivo murine 
model and in vitro RAW264.7 cells. Inflammatory cell infiltration, goblet cell hyperplasia, Th2 cytokines, the phosphorylation of JNK and ER stress markers were all significantly inhibited when CpG-ODN was given during the development of allergic airway inflammation. CpG-ODN may be introduced as an adjunctive therapy for allergic airway inflammation such as asthma.

\section{Introduction}

Asthma is a heterogeneous disease characterized by chronic airway inflammation involving multiple cells and cellular components, such as eosinophils, mast cells, T lymphocytes, neutrophils and airway epithelial cells. ${ }^{1,2}$ Allergic asthma is an important type of asthma, accounting for $60 \%$ to $80 \%$ of asthma., ${ }^{3,4}$ Anti-inflammatory and bronchodilator treatments are the main therapies for asthma, including inhaled corticosteroids, beta-agonists and systemic steroids in severe patients. ${ }^{4,5}$ However, about $5-10 \%$ of patients with asthma symptoms is poorly controlled by current strategies. ${ }^{6}$ And the side effects caused by the excessive use of corticosteroids are also the main factors that aggravate the burden of disease. ${ }^{7}$

Over the past few decades, the increased prevalence of allergic asthma is suggested to be due to environmental alterations, one hypothesis is the decreased microbe's exposure. Exposure in microbe-rich environment, for example rich of bacteria, the risk of allergic asthma sensitization is reduced. ${ }^{8}$ This is because of bacterial DNA, which contains unmethylated $\mathrm{CpG}$ motifs, has been conferred immunomodulatory properties. ${ }^{8}$ Synthetic CpGoligodeoxynucleotide (CpG-ODN), which can mimic the effect of bacterial DNA, reportedly binds to TLR9 receptor on B cells, dendritic cells and other types of cells, it induces Th1 response, and secretion of IFN- $\gamma$ and IL-12 which potently inhibit the expression of Th2 cytokines such as IL-4 and IL-5. ${ }^{9,10}$ These Th2 cytokines promote the chemotaxis and degranulation of eosinophils, mast cells and B cells, therefore contribute to $\operatorname{IgE}$ production. ${ }^{11}$ Unsurprisingly, exposure of mice to CpGODN is increasingly reported to reduce the risk of developing asthma in animal models. ${ }^{8,9,12}$ Moreover, as a TLR9 agonist and immune adjuvant, $\mathrm{CpG-ODN}$ has been shown efficacy in attenuating allergic disorder in human clinical trials. ${ }^{13}$ However, how CpG-ODN exerting these protective effects remains elusive and needs further investigation.

Under various exogenous and endogenous stimuli, unfolded and misfolded proteins will be largely produced. ${ }^{14}$ As we known, the biosynthesis, folding and structural maturation of more than a third of the proteins are occurred in the endoplasmic reticulum (ER). ${ }^{15}$ When unfolded and misfolded proteins accumulate in the ER above a critical threshold, ER stress will be induced, ${ }^{16}$ which is a hallmark of many inflammatory diseases. Then the unfolded protein response (UPR) signal transduction pathway is initiated to combat this ER stress. ${ }^{16}$ A growing number of studies have addressed that ER stress plays a vital role in many diseases, including neurodegenerative diseases, inflammatory diseases, metabolic diseases and malignant tumors. ${ }^{16}$ However, its role in allergic asthma, and the regulation of $\mathrm{CpG}-\mathrm{ODN}$ on ER stress are still unknown.

On the other hand, the c-Jun N-terminal kinase (JNK) signal, one of the stress-induced mitogen-activated protein kinase (MAPK) family members, is activated through phosphorylation cascades in response to various internal and external environmental stresses. ${ }^{17}$ Increased phosphorylation of JNK reportedly leads to ER stress in various diseases, such as inflammatory diseases ${ }^{18}$ and immune diseases. ${ }^{19}$ We and others studies have confirmed that the phosphorylation of JNK is significant increased when exposure to OVA, and its specific inhibitor SP600125 obviously suppresses mucus hypersecretion, inflammatory cell infiltration and production of inflammatory factors. $^{20,21}$ However, whether this OVA-induced JNK phosphorylation is associated with ER stress in allergic airway inflammation is unclear. Additionally, CpG-ODN is reported to regulate the activation of $\mathrm{MAPK},{ }^{22}$ and $\mathrm{CpG}$ ODN-FITC is found to co-localize with the ER stressassociated protein GRP78 as observed by confocal microscopy. $^{23}$ Therefore, we reasonably speculate that CpG-ODN may confer protection against airway inflammation via regulation of $\mathrm{JNK}$ activation and ER stress.

Moreover, in the pathogenesis of asthma, macrophages have been recognized more and more, especially alveolar macrophages (AMs). ${ }^{24}$ Under normal circumstances, AMs maintain lung homeostasis. ${ }^{25}$ When stimulated by various internal and external stimuli, they exhibits a proinflammatory phenotype. ${ }^{25}$ Moreover, in the pathogenesis of asthma, macrophages also act as antigen-presenting cells (APC) to produce enzymes, cytokines and chemokines which are closely related to the recruitment of inflammatory cells. $^{26,27}$ Importantly, endoplasmic reticulum stress (ER stress) is a critical mechanism of regulation of macrophage phenotype differentiation, ${ }^{28}$ its level in macrophages of asthmatic patients is higher than that of healthy people. ${ }^{29}$ 
In the present study, we aimed to investigate the effect of CpG-ODN on the ER stress in vivo in OVA-induced murine allergic airway inflammation and in vitro in OVAstimulated RAW264.7 cells, and reveal whether such effect of CpG-ODN is mediated by JNK signal pathway.

\section{Materials and Methods}

\section{Animals and Cells}

SPF C57BL/6 mice (Female, 5-week-old, 16-18g) were purchased from Anhui Laboratory Animal Center (Hefei, China). All mice were housed at a temperature of $20-26^{\circ} \mathrm{C}$ and on a $12 \mathrm{~h} \mathrm{light}$ and dark alternating time. This study was conducted in accordance with the Basel Declaration and the ethical guidelines by the International Council for Laboratory Animal Science (ICLAS). The Animal Care and Use Committee of Anhui Medical University approved all experimental procedures (Permit Number: LLSC20170361).

The murine macrophage cell line RAW264.7 was purchased from the Cell Bank of the Chinese Academy of Sciences (Shanghai, China) and cultured in Dulbecco's modified Eagle's medium (DMEM; Sigma Inc., St. Louis, MO), supplemented with $10 \%$ fetal bovine serum (FBS, Gibco, Grand Island, NY) and 1\% penicillin/streptomycin solution (C0222, Beyotime Biotechnology, China) at $37^{\circ} \mathrm{C}$ in $5 \%$ $\mathrm{CO}_{2}$. RAW264.7 cells were grown in 24-well plates to 70$80 \%$ confluence and then stimulated with OVA $(500 \mu \mathrm{g} / \mathrm{mL}$, Sigma). Some cells were treated with CpG-ODN 1668 (1.5 $\mu \mathrm{M}, 5 \mu \mathrm{M}$, Sangon, Shanghai, China) for $1 \mathrm{~h}$ prior to stimulation with OVA. Some cells were treated with SP600125 (10 $\mu \mathrm{M}$, Sigma Inc., St. Louis, MO) for 2 $\mathrm{h}$ prior to stimulation with OVA.

\section{Oligodeoxynucleotides}

The CpG-ODN 1668 (5'-TCC ATG ACG TTC CTG ATG CT-3') was synthesized and supplied by Sangon Biotech (Shanghai, China), CpG-ODN (30 $\mu \mathrm{g} /$ mice) was administrated by i.p. injection.

\section{OVA-Induced Acute Allergic Airway Inflammation Model and Treatments}

Acute allergic airway inflammation was induced according to our previous study and modified. ${ }^{30}$ On Day 0 and 7, mice were sensitized with $0.5 \mathrm{~mL}$ suspension consisting of

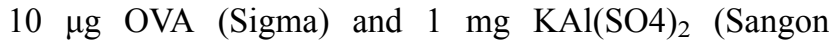
Biotech, Shanghai, China) in saline intraperitoneally. Then from Day 14 to 20, mice were challenged with $1 \%$ OVA by airway inhalation 30 min every day. The mice were randomly grouped into two parts. Part 1 comprised four groups (control, CpG-ODN, OVA, OVA + CpGODN), $\mathrm{n}=6$ for each; Part 2 comprised four groups (OVA, OVA + SP600125, OVA + CpG-ODN, OVA + CpG-ODN + SP600125), $\mathrm{n}=6$ for each. For CpG-ODN, OVA + CpG-ODN and OVA + CpG-ODN + SP600125 groups, $30 \mu \mathrm{g}$ of $\mathrm{CpG}-\mathrm{ODN}$ were given intraperitoneally to mice one hour before OVA challenge. For OVA + SP600125, OVA + CpG-ODN + SP600125 groups, SP600125 (30 mg/kg, Sigma) were given intraperitoneally to mice two hours before OVA challenge.

\section{Bronchoalveolar Lavage Fluid Collection and Analysis}

After $24 \mathrm{~h}$ of the last challenge, mice were sacrificed. Then the skin was cut open to expose trachea and thorax, and left lung lavage was performed five times on the ice with $0.3 \mathrm{~mL}$ phosphate buffered saline (PBS). The collected alveolar lavage fluid was centrifuged at $4^{\circ} \mathrm{C}, 1000 \mathrm{rpm}$ for $10 \mathrm{~min}$. Finally, the precipitate was used for cell counting and differentiation. The pellets were re-suspended for total cell counts using a hemocytometer. Wright-Giemsa staining was used for eosinophil counts in a blind manner.

\section{Histopathological Staining of the Lung Tissue}

In order to assess the degree of inflammation and mucus secretion around the bronchi, a lobe of the right lung was used for histopathology. It was first fixed with $4 \%$ paraformaldehyde, then dehydrated, embedded in paraffin, and cut into $4 \mu \mathrm{m}$ sections for hematoxylin-eosin staining (HE) and periodic acid-Schiff staining (PAS). The score of cell infiltration or goblet cell hyperplasia were assessed as previously reported. ${ }^{12}$

\section{Immunohistochemistry}

Briefly, tissue sections $(4 \mu \mathrm{m})$ were incubated with $\mathrm{F} 4 / 80$ (Santa Cruz, CA) primary antibody at $4{ }^{\circ} \mathrm{C}$ overnight. The next day, sections were incubated with HRP-conjugated secondary antibody (Beijing Golden Bridge Biotechnology, Beijing, China) for $1 \mathrm{~h}$ at $37^{\circ} \mathrm{C}$. After rinsed with PBS for three times, sections were stained by diaminobenzidine (DAB), and dark-brown staining was considered positive. The results were analyzed under a Carl Zeiss Axio Scope.A1 (Carl Zeiss, German). Representative images (magnification $\times 200$ ) were acquired. 


\section{Western Blotting}

The lung tissue was cut up on ice with RIPA buffer lysate and then homogenized by tissuelyser (JINGXIN, Shanghai, China) to extract the total protein. Equal amounts of lung homogenate or cell lysate were separated by a $12 \%$ SDS-PAGE and then transferred to PVDF membrane. The PVDF membrane was blocked in 5\% non-fat milk for $1 \mathrm{hr}$. The primary antibodies were as follow: IL-4, IL-5, IL-13, CHOP, XBP1, ATF6 $\alpha$ and GRP78 (Santa Cruz, CA). These antibodies were incubated at room temperature for $3 \mathrm{~h}$ and then overnight at $4^{\circ} \mathrm{C}$. The next morning, after washing with TBST for three times, the corresponding secondary antibody was incubated for 1 $\mathrm{h}$ and then washed with TBST for three times. Finally, Enhanced Chemiluminescence (ECL) Kit (Affinity, China) and Chemiluminescence imaging system (Qin Xiang, Shanghai, China) were used to observe the protein immunostaining.

\section{Immunofluorescence Staining and Confocal Microscopy}

$2.5 \times 10^{4}$ RAW264.7 cells were seeded on sterile glass cover slips in 12-well plates, after allowed to adhere to glass cover lips, cells were treated as described above and stimulated with OVA at $37^{\circ} \mathrm{C}$ for $24 \mathrm{~h}$. The cells were then fixed with $4 \%$ paraformaldehyde for $15 \mathrm{~min}$. Following fixation, the cells were permeabilized with $0.2 \%$ TritonX100 for $10 \mathrm{~min}$ at room temperature, blocked with $10 \%$ BSA for $15 \mathrm{~min}$ at $37{ }^{\circ} \mathrm{C}$. And then exposed to CHOP, XBP1, ATF6 $\alpha$ and GRP78 antibodies (1:100 dilutions) overnight at $4^{\circ} \mathrm{C}$. After washing, the cells were incubated with Alexa Fluor $594^{\circledR}$-conjugated anti-mouse fluorescent secondary antibodies (1:400 dilutions, Jackson ImmunoResearch Lab, Inc. West Grove, PA, USA) at 37 ${ }^{\circ} \mathrm{C}$ for $40 \mathrm{~min}$ in the dark and DAPI (C1002, Beyotime Biotechnology, China) for $8 \mathrm{~min}$ at room temperature. Finally, the cells were examined under a laser scanning confocal microscope. Fluorescence intensity of the images was analyzed with Leica Application Suite X System (Leica, Wetzlar, Hessen, GER).

\section{Statistic Analysis}

Data were expressed as mean \pm SEM (standard error of mean). Statistical comparisons were performed using oneway ANOVA with the Bonferroni test between groups. Statistical significance was set at $\mathrm{p}<0.05$.

\section{Results}

\section{CpG-ODN Alleviates OVA-Induced}

\section{Acute Allergic Airway Inflammation}

Wild-type (WT) C57 mice were used to establish the allergic airway inflammation model according to the protocol illustrated in Figure 1A. As compared to control mice, challenged with $1 \%$ OVA for 7 consecutive days induced a large amount of inflammatory cells infiltration and goblet cell hyperplasia in the airway as analyzed by $\mathrm{HE}$ and PAS staining of lung tissue section (Figure 1B and C). However, treatment with CpG-ODN (30 ug/mice/day) 1h before OVA inhalation for 7 consecutive days significantly inhibited OVA-induced inflammatory cells infiltration and $\mathrm{PAS}^{+}$cells (Figure 1B and C). Similarly, BALF smear staining showed that the effect of saline in control group did not induce eosinophil infiltration, but OVA challenge induced a large number of inflammatory cells infiltration in BALF (Figure 1D and E), particularly eosinophils, and these OVA-induced inflammatory cells accumulation was sharply suppressed by CpG-ODN treatment (Figure 1D and E). Consistently, the levels of Th2 cytokines such as IL-4, IL-5, IL-13 in the lung tissues were significantly reduced by daily treatment of CpG-ODN (Figure 1F and G).

\section{CpG-ODN Suppresses OVA-Induced Endoplasmic Reticulum Stress in the Airway and RAW264.7 Cells}

ER stress is increasingly suggested to be involved in the pathophysiology of allergic airway inflammation. ${ }^{31}$ In the present study, the levels of ER stress-associated protein CHOP, XBP1, ATF6 $\alpha$ and GRP78 in lung tissues were obviously increased in response to OVA challenge as compared to those of control mice (Figure 2A and B). However, such OVA-induced ER stress was profoundly inhibited by administration of CpG-ODN (Figure 2A and B). In addition, the imbalance of UPR caused by ER stress severely affects the function of macrophages which play an important role in allergic airway inflammation. ${ }^{32}$ So we examined the changes of macrophages in the lung between control and OVAchallenged mice by immunohistochemistry. We found that compared with control mice, significant infiltration of macrophages in the lung was found after challenged by OVA (Figure S1). Consistently, the expressions of these four ER stress markers in RAW264.7 macrophage cells were significantly increased after OVA stimulation, but 
A

$$
\begin{aligned}
\text { Time } & \text { (Days) } \\
\uparrow & \uparrow
\end{aligned}
$$

D
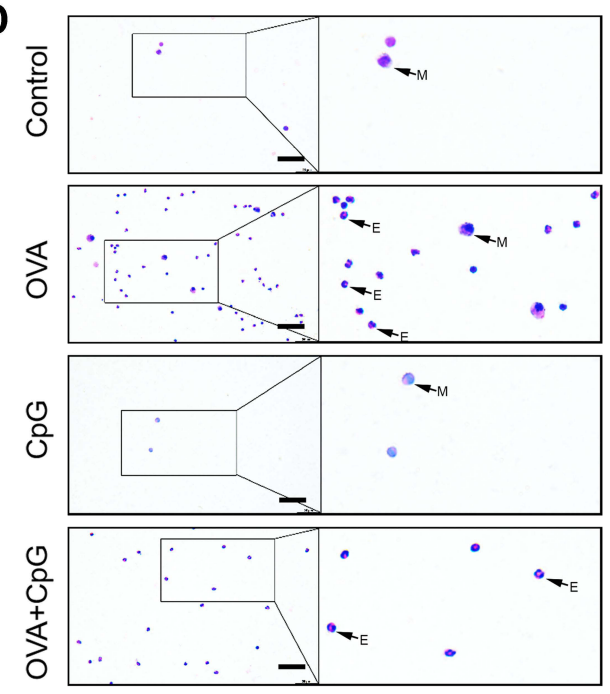

E

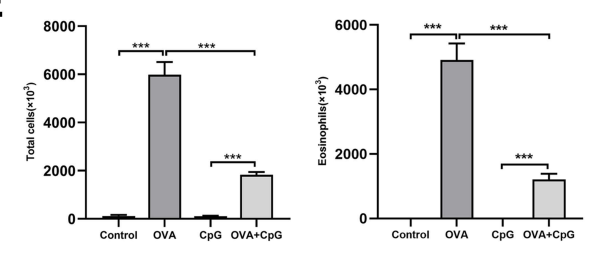

B

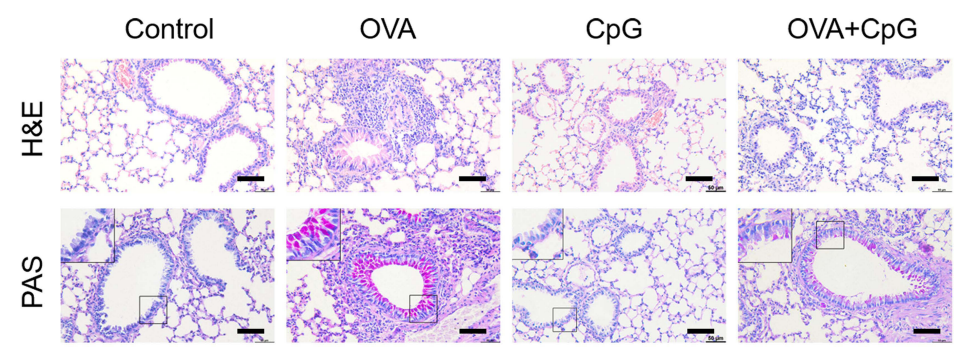

C
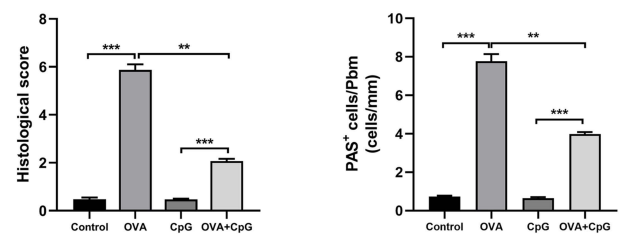

F

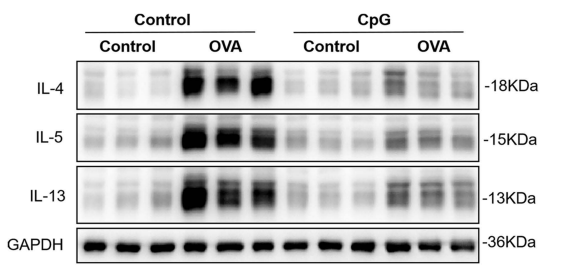

G

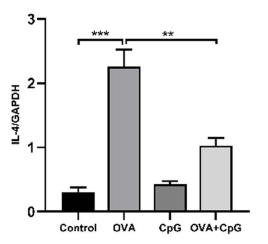

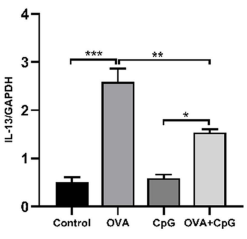

Figure I CPG-ODN ameliorates OVA-induced allergic airway inflammation. (A) Protocol of establishing murine allergic airway diseases model treated with or without $C_{P G-O D N}$. Mice were sensitized on Day 0 and 7; challenged from Day 14 to 20 by OVA. Mice in control group were sensitized and challenged with saline. Mice in CpGODN group were sensitized and challenged with saline and administrated intraperitoneally with $30 \mu g / m i c e$ every day for 7 days. Mice in OVA group were sensitized and challenged with OVA. Mice in OVA + CPG-ODN group were sensitized and challenged with OVA and administrated intraperitoneally with $30 \mu \mathrm{g} / \mathrm{mice}$ every day for 7 days. (B) Lung sections from control, OVA, CpG-ODN and OVA + CpG-ODN group were stained with H\&E to analyze cell infiltration or periodic acid-Schiff (PAS) to evaluate mucus production. The scale bar is $50 \mu \mathrm{m}$. (C) The extent of peribronchial inflammation stained by HE staining, airway mucus secretion and goblet cell hyperplasia stained by periodic acid-Schiff (PAS) staining method was scored. (D) Representative stained BALF cell smears and BALF cellularity from control, OVA, CPG-ODN and OVA + CPGODN group, the cells were isolated and stained with Wright-Giemsa. E-eosinophil, M-monocyte. The scale bar is $25 \mu \mathrm{m}$. (E) Quantification of the level of total cells and Eosinophils in BALF. (F) Representative blots showed the expression of Th2 associated cytokines such as IL-4, IL-5 and IL-I 3 in lung extracts. (G) Quantification of the level of IL-4, IL-5 and IL-13. Data were expressed as mean \pm SEM of six mice per group. $*_{p}<0.05, * * p<0.01, * * * p<0.00$ I.

such OVA-induced expression was notably decreased after the treatment of $1.5 \mu \mathrm{M}$ and $5 \mu \mathrm{M}$ CpG-ODN (Figure 2C), and there is no statistical difference between these two doses (Figure 2D). Meanwhile, we also revealed that CpG-ODN markedly reduced the immunostaining intensity of $\mathrm{CHOP}$, XBP1, ATF6 $\alpha$ and GRP78 (red fluorescence, Alexa Fluor ${ }^{\circledR}$ 594) after exposure to OVA for $24 \mathrm{~h}$ in RAW264.7 macrophage cells (Figure 2E). These results indicated that CpGODN potentially inhibited ER stress in OVA-induced acute allergic airway inflammation and RAW264.7 cells.

\section{The Effect of CpG-ODN on OVA-Induced $\mathrm{p}$-JNK Expression}

It has been reported that ER stress is accompanied by the activation of MAPK, ${ }^{33}$ and the phosphorylation of JNK is associated with tissue inflammation and the production of pro-inflammatory factors. ${ }^{34,35}$ Here, we found that compared to control mice, the expression of p-JNK increased significantly in response to OVA challenge, but the level of total JNK was not affected (Figure 3A and B). While administration of $\mathrm{CpG}-\mathrm{ODN}$, the phosphorylation level 
A

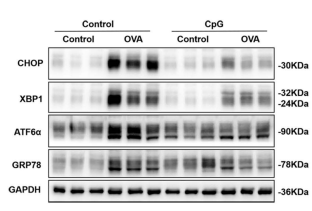

B
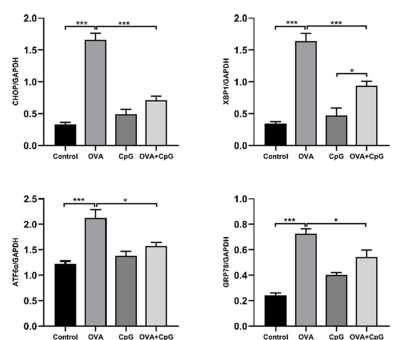

C

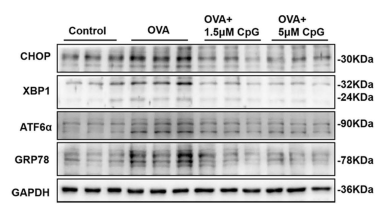

D

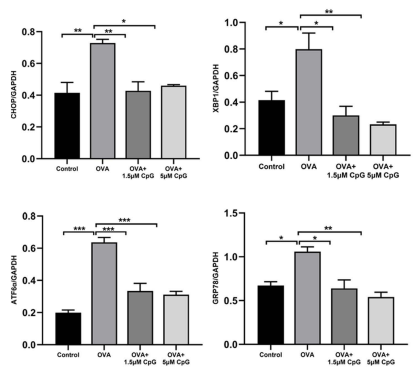

$\mathbf{E}$
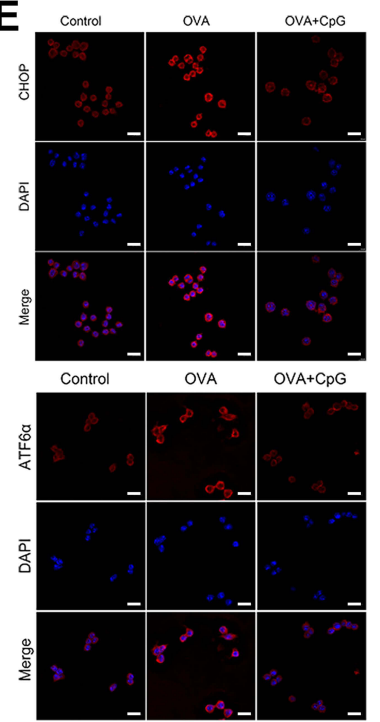
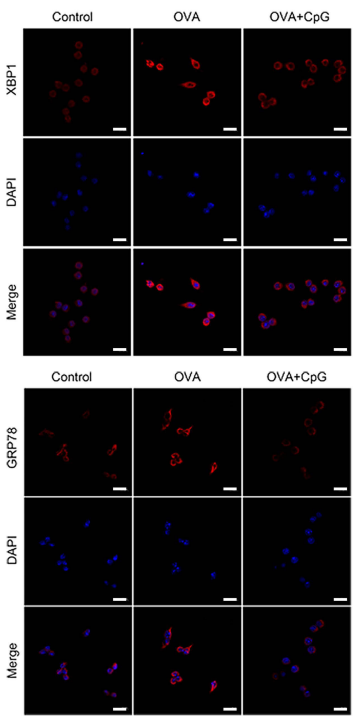

Figure 2 CPG-ODN suppresses OVA-induced endoplasmic reticulum stress. (A) Representative blots showing the protein levels of CHOP, XBPI, ATF6 $\alpha$ and GRP78 in mice from control, OVA, CpG-ODN and OVA + CpG-ODN group. (B) Quantification of the expression of CHOP, XBPI, ATF6 $\alpha$ and GRP78 as referencing to GAPDH in lung extract. (C) Representative blots showing the expression of CHOP, XBPI, ATF6 $\alpha$ and GRP78 in RAW264.7 from control, OVA, OVA + I.5 $\mu$ M CPG-ODN and OVA + $5 \mu \mathrm{M}$ CpG-ODN group. (D) Quantification of the expression of CHOP, XBPI, ATF6 $\alpha$ and GRP78 as referencing to GAPDH in RAW264.7 cells. (E) By using immunofluorescence, the expression of CHOP, XBPI, ATF6 $\alpha$ and GRP78 in RAW264.7 was detected by confocal microscopy (magnification $\times 63$ oil). Nuclei were stained by DAPI. The scale bar is $10 \mu \mathrm{m}$. Data were expressed as mean \pm SEM of six mice per group or three experiments for RAW264.7 cells. $* p<0.05$, $* * p<0.0$ I, $* * * p<0.00$ I.

of JNK was obviously decreased, and it had no effect on the level of total JNK (Figure 3A and B). Similarly, the same results were observed in RAW264.7 cells (Figure 3C and D). These results definitely showed that CpG-ODN suppressed OVA-induced JNK phosphorylation in vivo and in vitro.

\section{The Effect of JNK Inhibitor SP600I 25 on OVA-Induced Allergic Airway Inflammation}

To further confirm whether CpG-ODN alleviates allergic airway inflammation by suppressing OVA-induced JNK phosphorylation, we used SP600125 (an antagonist of JNK) to block the phosphorylation of JNK. Firstly, in animal and cell models, we found that after treatment with SP600125, the OVA-induced expression of p-JNK was almost suppressed (Figure 4A and B), and there is no statistical difference among SP600125, CpG-ODN and CpG-ODN + SP600125 group (Figure 4C and D). Then we demonstrated that compared to the OVA group, administration of SP600125 clearly inhibited inflammatory cells infiltration and PAS $^{+}$cells in the lung tissue by HE and PAS staining (Figure 5A and B). Similarly, BALF smear staining showed that after treatment with SP600125 significantly reduced the infiltration of inflammatory cells, especially eosinophil
(Figure 5C and D). The pathological features was not significant difference between CpG-ODN group and SP600125 group. And CpG-ODN plus SP600125 treatment did not further reduce the infiltration of inflammatory cells and the secretion of mucus with comparison to that of $\mathrm{CpG}-\mathrm{ODN}$ or SP600125 single treatment.

Additionally, the expression of Th2 cytokines (IL-4, IL-5 and IL-13) in lung extracts was markedly decreased by SP600125, CpG-ODN or CpG-ODN plus SP600125 with comparison to that of the OVA group (Figure $5 \mathrm{E}$ and F), but the Th2 cytokine levels in SP600125 group was higher than that of CpG-ODN group or CpG-ODN + SP600125 group, suggesting such inhibitory effect of SP600125 on Th2 cytokine production was weaker than CpG-ODN (Figure 5E and F). Thus, these results implied that CpG-ODN may exert its anti-inflammatory effect partly through the JNK pathway in OVA-induced allergic airway inflammation model.

\section{The Effect of SP600I25 on OVA-Induced ER Stress}

Many studies verified that phosphorylation of JNK is involved in ER stress. ${ }^{36}$ Therefore, we investigated whether the effect of CpG-ODN on ER stress in the OVA-induced allergic airway inflammation was mediated by JNK signal 

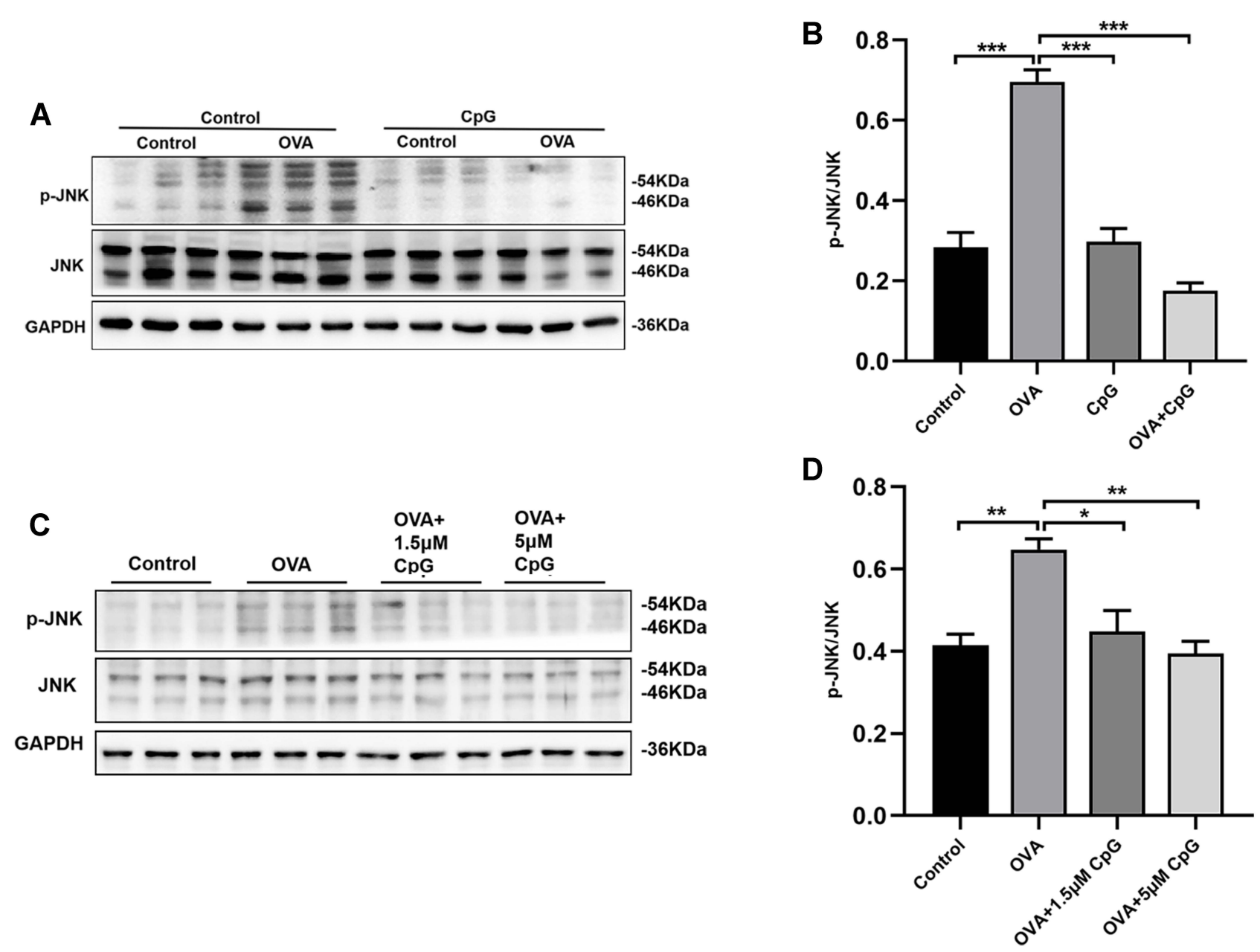

Figure 3 The effect of CPG-ODN on OVA-induced p-JNK expression. (A) Representative blots showing the expression of p-JNK and JNK in mice from control, OVA, CpG-ODN and OVA + CpG-ODN group. (C) Representative blots showing the expression of p-JNK and JNK in RAW264.7 cells from control, OVA, OVA + I.5 $\mu$ M CpGODN and OVA + 5 $\mu$ M CPG-ODN group. (B and $\mathbf{D})$ Quantification of the expression of p-JNK and JNK as referencing to GAPDH in mice and RAW264.7 cells. Data were expressed as mean \pm SEM of six mice per group or three experiments for RAW264.7 cells. $*_{p}<0.05, *^{*} p<0.01, *^{* *} p<0.00 \mathrm{I}$.

pathway. We found that OVA-induced protein expressions of CHOP, XBP1, ATF6 $\alpha$ and GRP78 were obviously reduced in lung tissues by SP600125 treatment (Figure 6A and B). Moreover, CpG-ODN or CpG-ODN plus SP600125 treatment significantly suppressed the OVA-induced ER stress, and their inhibitory effect was more effective than SP600125 (Figure 6A and B). The similar results were demonstrated in RAW264.7 cells by immunoblotting (Figure 6C and D). Furthermore, we found that compared to OVA-stimulated RAW264.7 macrophage cells, the intensity of CHOP, XBP1, ATF6 $\alpha$ and GRP78 (red fluorescence, Alexa Fluor ${ }^{\circledR}$ 594) were also apparently weaker in SP600125, CpG-ODN and CpG-ODN plus SP600125 group (Figure 6E).

\section{Discussion}

We have previously shown that $\mathrm{CpG}-\mathrm{ODN}$ protected against OVA-induced allergic airway inflammation, which was associated with the inactivation of NLRP3 inflammasome. ${ }^{12}$ In the present study, we further revealed that CpG-ODN exerted protective effect on OVA-induced allergic airway inflammation by inhibiting ER stress, and suggested that the underlying mechanism may be associated with the suppression of JNK signal by using in vivo murine model and in vitro RAW264.7 cells. This study thus provides another possible mechanism, ie, suppression of JNK-mediated ER stress, by which CpG-ODN protects from allergic airway inflammation.

Synthesized oligonucleotides such as CpG-ODN which containing bacterial unmethylated cytosine-guanine dinucleotide sequence, ${ }^{7}$ have been widely used to prevent or treat malignant tumor, infectious diseases and allergic diseases. ${ }^{13}$ According to the difference of structure and immune response, $\mathrm{CpG}-\mathrm{ODN}$ can be divided into class-A (referred to $\mathrm{D}$ type ODN), class-B (referred to $\mathrm{K}$ type 

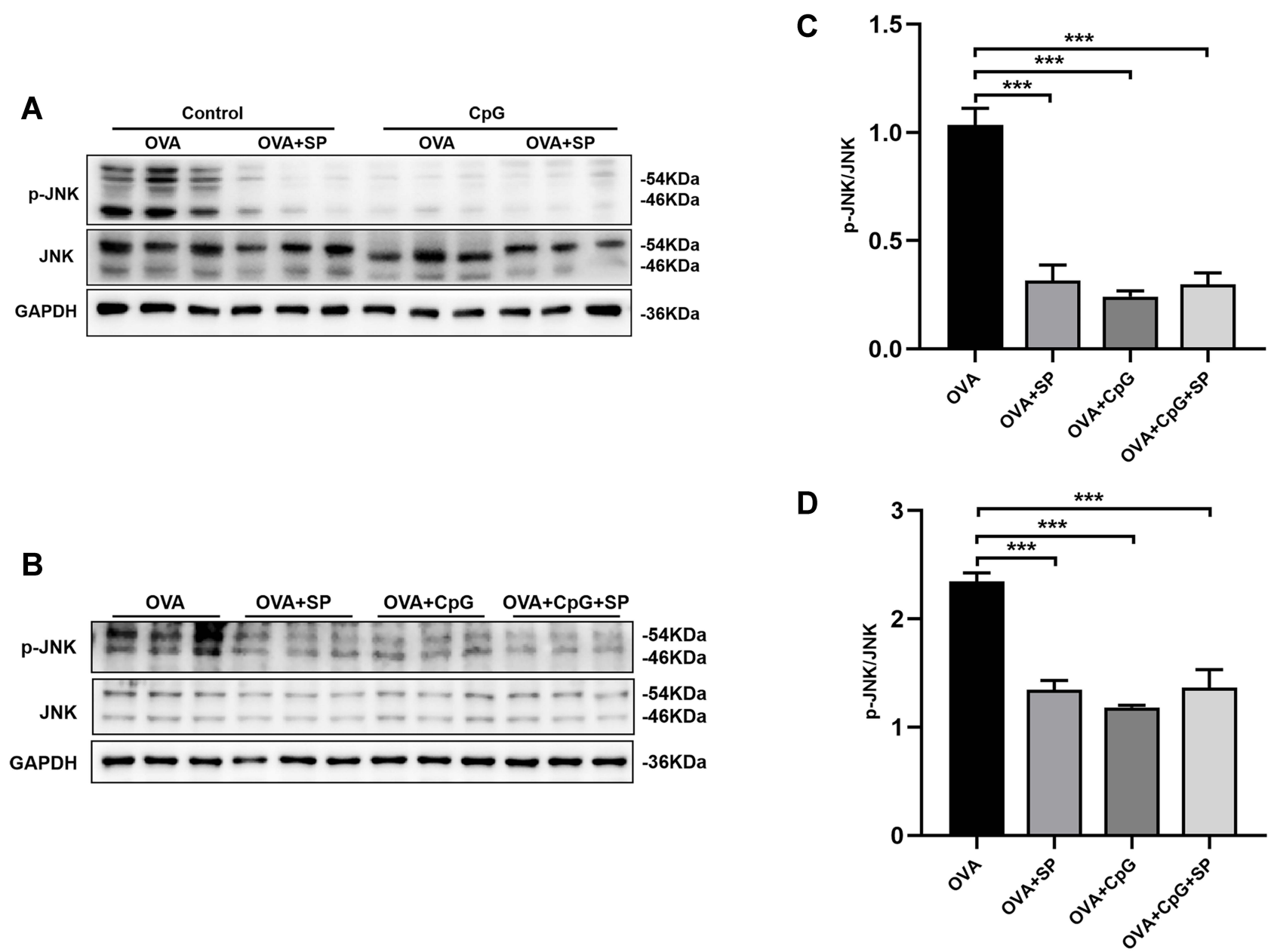

Figure 4 The effect of SP600I25 on OVA-induced p-JNK expression. (A and B) Representative blots showing the expression of p-JNK and JNK in mice and RAW264.7 cells from OVA, OVA + SP600125, OVA + CPG-ODN and OVA + CPG-ODN + SP600I25 group. (C and D) Quantification of the expression of p-JNK and JNK as referencing to GAPDH in mice and RAW264.7 cells. Data were expressed as mean \pm SEM of six mice per group or three experiments for RAW264.7 cells. ***p $<0.001$.

ODN), class-C and class-P. ${ }^{37,38}$ In our current study, we used the fully phosphorothioated CpG-ODN, a type B CpG-ODN, which has been reported to powerfully inhibit the Th2 response and is most commonly used in clinical trials. ${ }^{13}$ The results in this study showed that CpGODN reduced the protein levels of Th2 type cytokines (IL4, IL-5 and IL-13) and airway inflammation, which were consistent with our previous research. ${ }^{12}$ The present study together with our previous findings ${ }^{12}$ confirmed that $\mathrm{CpG}$ ODN exerted protective role in controlling allergic airway inflammation.

Inhaled environmental allergens reportedly trigger ER stress and ER homeostasis dysregulation, ${ }^{39}$ prolonged ER stress and UPR consequently induce persistence of airway inflammation. Therefore, ER stress, which ultimately resulted in activation of unfolded protein response (UPR), has been demonstrated in the pathogenesis of allergen-induced asthma. ${ }^{40}$ Blocking ER stress by inhibitor
4PBA could alleviate allergic airway inflammation. ${ }^{41}$ Under normal circumstances, the three transmembrane proteins of ATF6, IRE1 and PERK on the ER membrane act as receptors of the ER and tightly bind to GRP78 to maintain the homeostasis state of ER. ${ }^{42}$ Once stressed, misfolded proteins accumulate in the cavity of ER, and GRP78 dissociates from the transmembrane proteins, leading to GRP78, ATF6, IRE1 and PERK activation. ${ }^{42}$ In addition, persistent ER stress induces the up-regulation of CHOP through PREK, leading to cell apoptosis, ${ }^{43}$ and IRE1 induces XBP1 to increase the production of proinflammatory cytokines and mucus secretion-related factors. ${ }^{44}$ In the present study, we revealed increased protein expression of CHOP, XBP1, ATF6 $\alpha$ and GRP78 in OVA-induced allergic airway inflammation and in OVAstimulated RAW264.7 macrophage cells, which was obviously reduced by $\mathrm{CpG}-\mathrm{ODN}$ treatment. These findings indicated that the vital role of $\mathrm{CpG}-\mathrm{ODN}$ in maintaining 
A
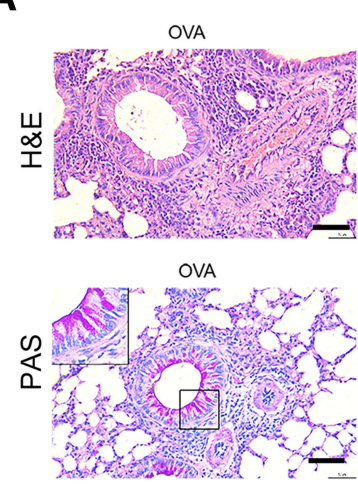

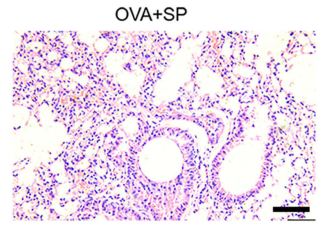

$O V A+S P$

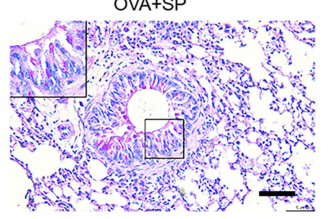

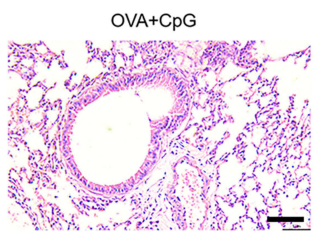

OVA+CpG

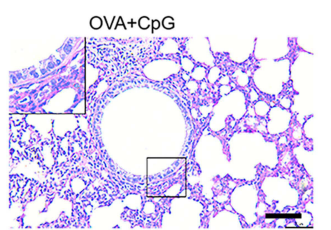

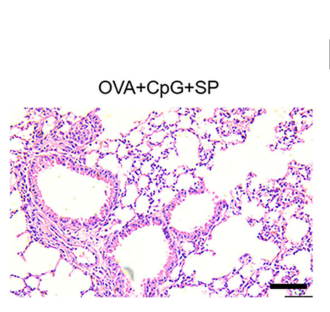

$\mathrm{OVA}+\mathrm{CpG}+\mathrm{SP}$

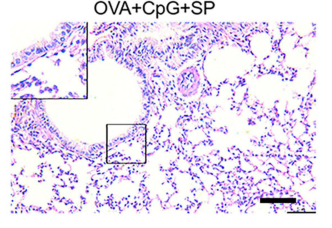

D

C
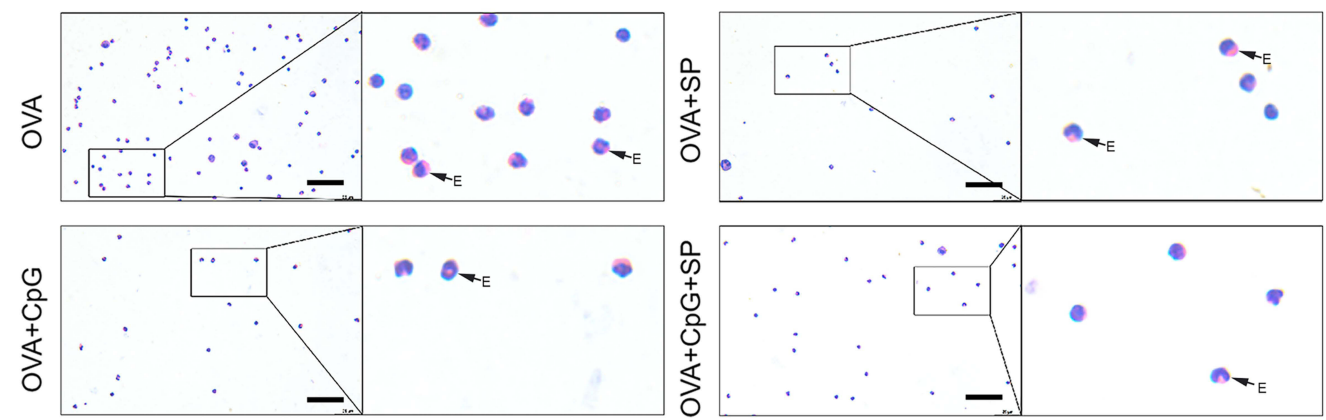

E

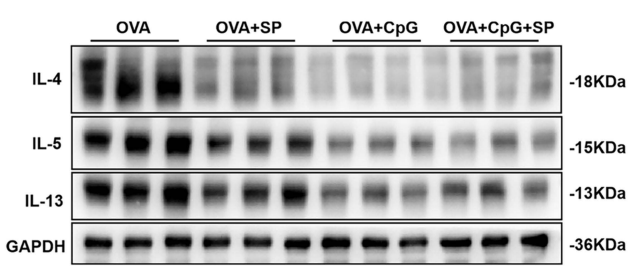

$\mathbf{F}$

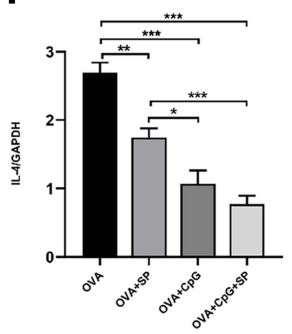

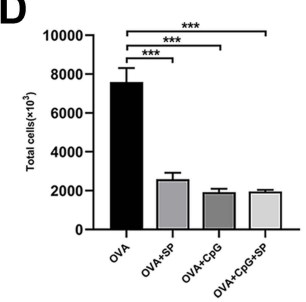
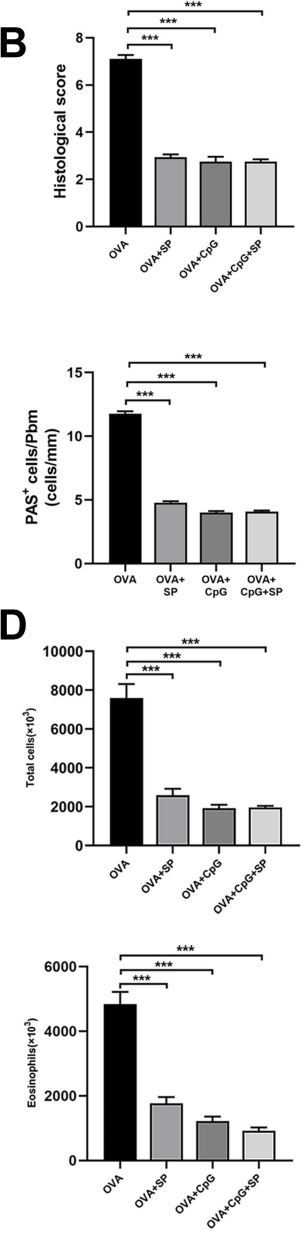

Figure 5 The effect of JNK antagonist SP600I25 on OVA-induced allergic airway inflammation. (A) Lung sections from OVA, OVA + SP600I25, OVA + CPG-ODN and OVA + CPG-ODN + SP600I25 group were stained with H\&E to analyze cell infiltration and periodic acid-Schiff (PAS) to evaluate mucus production. The scale bar is $50 \mu$ m. (B) The extent of peribronchial inflammation stained by HE, airway mucus secretion and goblet cell hyperplasia stained by periodic acid-Schiff (PAS) staining were scored. (C) Representative stained BALF cell smears and BALF cellularity from OVA, OVA + SP600I25, OVA + CpG-ODN and OVA + CPG-ODN + SP600I25 group. E-eosinophil. The scale bar is $25 \mu \mathrm{m}$. (D) Quantification of the level of total and Eosinophils in BALF. (E) Representative blots showed the expression of IL-4, IL-5 and IL-I 3 in lung extracts. (F) Quantification of the level of IL-4, IL-5, and IL-I 3 referencing to GAPDH. Data were expressed as mean \pm SEM of six mice per group. ${ }^{*} p<0.05$, $* * p<0.0$ I, *** $p<0.00$ I.

ER homeostasis and inhibiting ER stress in allergic airway inflammation.

However, the underlying mechanism of CpG-ODN attenuating OVA-induced ER stress remains unknown. As one of the key members of the MAP kinase family, JNK signal has been reported to mediate a variety of cellular functions through phosphorylation cascades. ${ }^{17}$ The activation of JNK signal is closely related to ER stress $^{20,21}$ and contributes to allergic airway inflammation. ${ }^{35}$ Blocking JNK phosphorylation by its specific inhibitor SP600125 markedly alleviates allergic airway inflammation. ${ }^{45,46}$ In the current study, we found that the expression of $\mathrm{p}$-JNK was highly upregulated after OVA challenged, which was in lined with our previous study. ${ }^{20,46}$ Furthermore, we demonstrated that such OVAinduced JNK phosphorylation was completely abolished 

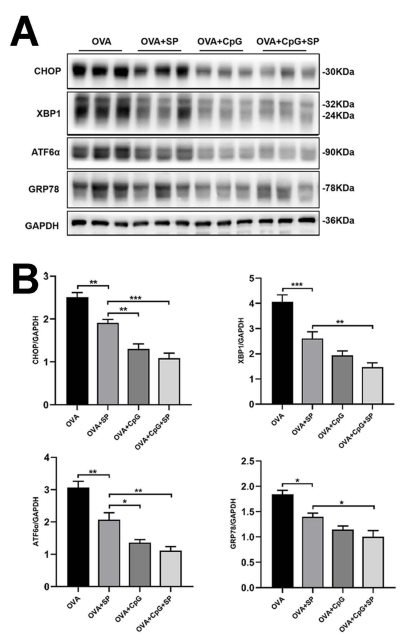

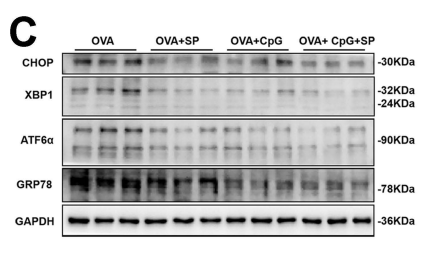

D
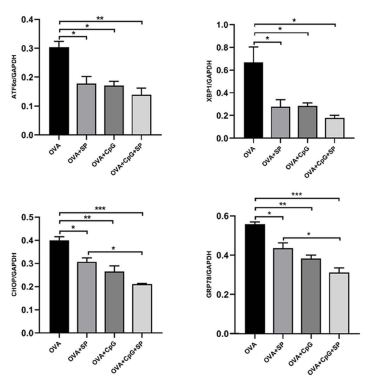
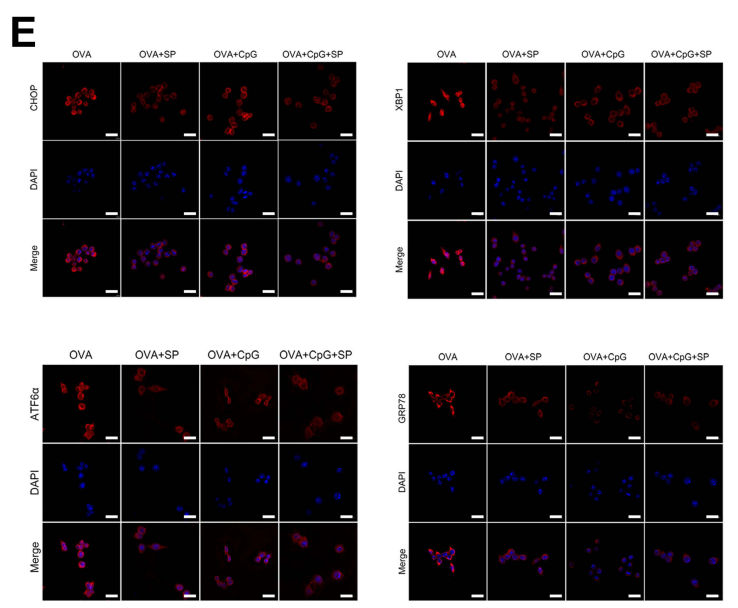

Figure 6 The effect of SP600I 25 on OVA-induced ER stress. (A and C) Representative blots showing the protein level of CHOP, XBPI, ATF6 $\alpha$ and GRP78 in mice and RAW264.7 cells from OVA, OVA + SP600I25, OVA + CpG-ODN and OVA + CPG-ODN + SP600I 25 group. (B and D) Quantification of the expression of CHOP, XBPI, ATF6 $\alpha$ and GRP78 as referencing to GAPDH. (E) By using immunofluorescence, the expression of CHOP, XBPI, ATF6 $\alpha$ and GRP78 in RAW264.7 was detected by confocal microscopy (magnification $\times 63$ oil). Nuclei were stained by DAPI. The scale bar is $10 \mu \mathrm{m}$. Data were expressed as mean \pm SEM of six mice per group or three experiments for RAW264.7 cells. ${ }^{*} p<0.05,{ }^{* *} p<0.01$, $* * * p<0.001$.

by treatment of $\mathrm{CpG}-\mathrm{ODN}$ or SP600125 or CpG-ODN plus SP600125. However, although the expression levels of Th2 type inflammatory factors in lung tissue was notably reduced by SP600125 as compared to OVA group, they were still higher than that in the CpG-ODN and CpGODN plus SP60015 treatment group. Similarly, SP600125 significantly inhibited the protein expression of CHOP, XBP1, ATF6 $\alpha$ and GRP78 in lung extract and RAW264.7 cells compared with OVA group, but its such inhibitory effect was weaker than $\mathrm{CpG}-\mathrm{ODN}$ or $\mathrm{CpG}$ ODN plus SP600125. This is may be due to that the other two members of MAP kinase family, P38 and ERK MAP kinase, were also phosphorylated in the pathophysiology of asthma. ${ }^{4-50}$ Therefore, it is likely that CpGODN suppresses ER stress partly through JNK signal in OVA-induced allergic airway inflammation. It is noticeable that, in our present study, inhibition of JNK signal suppressed ER stress in allergic airway inflammation, but it has been previously reported that inhibition of ER stress notably decrease the expression of p-JNK. ${ }^{51}$ We speculated that the occurrence of ER stress induced by allergen stimulation, in turn, further increase the expression of p-JNK and form a vicious circle, thus aggravating allergic airway inflammation.

\section{Conclusion}

In summary, our study firstly demonstrated that CpG-ODN alleviates OVA-induced allergic airway inflammation through inhibiting on ER stress, and such effect of CpG-
ODN may be associated with its inhibitory effect on JNK signal in vivo murine model and in vitro RAW264.7 cells. CpG-ODN thus may be introduced as an adjunctive therapy for allergic airway inflammation such as asthma.

\section{Acknowledgments}

This work was supported by grants from the National Natural Science Foundation of China (No. 81770032), Foundation for Distinguished Young Scholars of the First Affiliated Hospital of Anhui Medical University, Program for the Youth Distinguished Talents of Anhui Medical University, and Key Lab of Geriatric Molecular Medicine of Anhui Province.

\section{Disclosure}

The authors report no conflicts of interest in this work.

\section{References}

1. Hammad H, Lambrecht BN. The basic immunology of asthma. Cell. 2021;184(9):2521-2522. doi:10.1016/j.cell.2021.04.019

2. Rothe T, Spagnolo P, Bridevaux PO, et al. Diagnosis and management of asthma - The Swiss guidelines. Respiration. 2018;95(5):364-380. doi:10.1159/000486797

3. Respiratory Allergy Group of Chinese Society of Allergy; Asthma Group of Chinese Thoracic Society, Chinese Medical Association. [Chinese guidelines for the diagnosis and treatment of allergic asthma (2019, the first edition)]. Zhonghua Nei Ke Za Zhi. 2019;58 (9):636-655. Chinese.

4. Papi A, Brightling C, Pedersen SE, Reddel HK. Asthma. Lancet. 2018;391(10122):783-800. doi:10.1016/S0140-6736(17)33311-1

5. Alwarith J, Kahleova H, Crosby L, et al. The role of nutrition in asthma prevention and treatment. Nutr Rev. 2020;78(11):928-938. doi:10.1093/nutrit/nuaa005 
6. El-Husseini ZW, Gosens R, Dekker F, Koppelman GH. The genetics of asthma and the promise of genomics-guided drug target discovery. Lancet Respir Med. 2020;8(10):1045-1056. doi:10.1016/S22132600(20)30363-5

7. Cevhertas L, Ogulur I, Maurer DJ, et al. Advances and recent developments in asthma in 2020. Allergy. 2020;75(12):3124-3146. doi:10.1111/all.14607

8. Sabatel C, Radermecker C, Fievez L, et al. Exposure to bacterial CpG DNA protects from airway allergic inflammation by expanding regulatory lung interstitial macrophages. Immunity. 2017;46(3):457-473. doi:10.1016/j.immuni.2017.02.016

9. Kim DH, Sohn JH, Park HJ, Lee JH, Park JW, Choi JM. CpG oligodeoxynucleotide inhibits cockroach-induced asthma via induction of IFN-gamma $(+)$ Th1 cells or Foxp3 $(+)$ regulatory T cells in the lung. Allergy Asthma Immunol Res. 2016;8(3):264-275. doi:10.4168/ aair.2016.8.3.264

10. Cowdery JS, Chace JH, Yi AK, Krieg AM. Bacterial DNA induces $\mathrm{NK}$ cells to produce IFN-gamma in vivo and increases the toxicity of lipopolysaccharides. J Immunol. 1996;156(12):6.

11. Fonseca DE, Kline JN. Use of CpG oligonucleotides in treatment of asthma and allergic disease. Adv Drug Deliv Rev. 2009;61 (3):256-262. doi:10.1016/j.addr.2008.12.007

12. Wu HM, Xie QM, Zhao CC, Xu J, Fan XY, Fei GH. Melatonin biosynthesis restored by $\mathrm{CpG}$ oligodeoxynucleotides attenuates allergic airway inflammation via regulating NLRP3 inflammasome. Life Sci. 2019;239:117067. doi:10.1016/j.1fs.2019.117067

13. Hanagata N. CpG oligodeoxynucleotide nanomedicines for the prophylaxis or treatment of cancers, infectious diseases, and allergies. Int J Nanomedicine. 2017;12:515-531. doi:10.2147/IJN.S114477

14. Chen X, Cubillos-Ruiz JR. Endoplasmic reticulum stress signals in the tumour and its microenvironment. Nat Rev Cancer. 2021;21 (2):71-88. doi:10.1038/s41568-020-00312-2

15. Anelli T, Sitia R. Protein quality control in the early secretory pathway. EMBO J. 2008;27(2):315-327. doi:10.1038/sj.emboj.7601974

16. Oakes SA, Papa FR. The role of endoplasmic reticulum stress in human pathology. Annu Rev Pathol. 2015;10:173-194. doi:10.1146/ annurev-pathol-012513-104649

17. Khorasanizadeh M, Eskian M, Gelfand EW, Rezaei N. Mitogenactivated protein kinases as therapeutic targets for asthma. Pharmacol Ther. 2017;174:112-126. doi:10.1016/j.pharmthera.2017. 02.024

18. Maeyashiki C, Melhem H, Hering L, et al. Activation of $\mathrm{pH}$-sensing receptor OGR1 (GPR68) induces ER stress via the IRE1 $\alpha / \mathrm{JNK}$ pathway in an intestinal epithelial cell model. Sci Rep. 2020;10(1):1438. doi:10.1038/s41598-020-57657-9

19. Ren Z, Chen S, Qing T, et al. Endoplasmic reticulum stress and MAPK signaling pathway activation underlie leflunomide-induced toxicity in HepG2 Cells. Toxicology. 2017;392:11-21. doi:10.1016/j. tox.2017.10.002

20. Wu HM, Fang L, Shen QY, Liu RY. SP600125 promotes resolution of allergic airway inflammation via TLR9 in an OVA-induced murine acute asthma model. Mol Immunol. 2015;67(2Pt B):311-316. doi:10.1016/j.molimm.2015.06.016

21. Santana FPR, da Silva RC, Ponci V, et al. Dehydrodieugenol improved lung inflammation in an asthma model by inhibiting the STAT3/SOCS3 and MAPK pathways. Biochem Pharmacol. 2020;180:114175. doi:10.1016/j.bcp.2020.114175

22. Kim JM, Kim NI, Oh YK, Kim YJ, Youn J, Ahn MJ. CpG oligodeoxynucleotides induce IL-8 expression in CD34+ cells via mitogen-activated protein kinase-dependent and NF-kappaBindependent pathways. Int Immunol. 2005;17(12):1525-1531. doi:10.1093/intimm/dxh345

23. Moon HG, Qin Z, Quan T, Xie L, Dela Cruz CS, Jin Y. Matrix protein $\mathrm{CCN} 1$ induced by bacterial DNA and $\mathrm{CpG}$ ODN limits lung inflammation and contributes to innate immune homeostasis. Mucosal Immunol. 2015;8(2):243-253. doi:10.1038/mi.2014.62
24. Saradna A, Do DC, Kumar S, Fu QL, Gao P. Macrophage polarization and allergic asthma. Transl Res. 2018;191:1-14. doi:10.1016/j. trsl.2017.09.002

25. Draijer C, Peters-Golden M. Alveolar macrophages in allergic asthma: the forgotten cell awakes. Curr Allergy Asthma Rep. 2017;17(2):12. doi:10.1007/s11882-017-0681-6

26. Fricker M, Gibson PG. Macrophage dysfunction in the pathogenesis and treatment of asthma. Eur Respir J. 2017;50(3):1700196. doi:10.1183/13993003.00196-2017

27. von Bubnoff D, Geiger E, Bieber T. Antigen-presenting cells in allergy. J Allergy Clin Immunol. 2001;108(3):329-339. doi:10.1067/ mai.2001.117457

28. Oh J, Riek AE, Weng S, et al. Endoplasmic reticulum stress controls M2 macrophage differentiation and foam cell formation. $J$ Biol Chem. 2012;287(15):11629-11641. doi:10.1074/jbc. M111.338673

29. Wang Y, Zhu J, Zhang L, et al. Role of C/EBP homologous protein and endoplasmic reticulum stress in asthma exacerbation by regulating the IL-4/signal transducer and activator of transcription 6/transcription factor EC/IL-4 receptor alpha positive feedback loop in M2 macrophages. J Allergy Clin Immunol. 2017;140(6):1550-1561 e1558. doi:10.1016/j.jaci.2017.01.024

30. Zhao CC, Xu J, Xie QM, Fan XY, Fei GH, Wu HM. Apolipoprotein E negatively regulates murine allergic airway inflammation via suppressing the activation of NLRP3 inflammasome and oxidative stress. Int Immunopharmacol. 2020;81:106301. doi:10.1016/j. intimp.2020.106301

31. Lee KS, Jeong JS, Kim SR, et al. Phosphoinositide 3-kinase-delta regulates fungus-induced allergic lung inflammation through endoplasmic reticulum stress. Thorax. 2016;71(1):52-63. doi:10.1136/ thoraxjnl-2015-207096

32. Díaz-Bulnes P, Saiz ML, López-Larrea C, et al. Regulator of macrophage polarization. Front Immunol. 2020;10:2951. doi:10.3389/ fimmu.2019.02951

33. Kong FJ, Ma LL, Guo JJ, Xu LH, Li Y, Qu S. Endoplasmic reticulum stress/autophagy pathway is involved in diabetes-induced neuronal apoptosis and cognitive decline in mice. Clin Sci. 2018;132 (1):111-125. doi:10.1042/CS20171432

34. Bin YF, Ma N, Lu YX, et al. Erythromycin reverses cigarette smoke extract-induced corticosteroid insensitivity by inhibition of the JNK/ c-Jun pathway. Free Radic Biol Med. 2020;152:494-503. doi:10.1016/j.freeradbiomed.2019.11.020

35. Athari SS. Targeting cell signaling in allergic asthma. Signal Transduct Target Ther. 2019;4:45.

36. Hotamisligil GS, Davis RJ. Cell signaling and stress responses. Cold Spring Harb Perspect Biol. 2016;8(10):a006072. doi:10.1101/cshperspect.a006072

37. Gürsel MG, Verthelyi D, Gürsel IG, Ishii KJ, Klinman DM. Differential and competitive activation of human immune cells by distinct classes of $\mathrm{CpG}$ oligodeoxynucleotide. J Leukoc Biol. 2002;71:813-820.

38. Kayraklioglu N, Horuluoglu B, Klinman DM. CpG oligonucleotides as vaccine adjuvants. In: Sousa Â, editor. DNA Vaccines: Methods and Protocols. New York, NY: Springer US; 2021;2197:51-85. doi:10.1007/978-1-0716-0872-2_4

39. Osorio F, Lambrecht B, Janssens S. The UPR and lung disease. Semin Immunopathol. 2013;35(3):293-306. doi:10.1007/s00281013-0368-6

40. Kim SR, Lee YC. Endoplasmic reticulum stress and the related signaling networks in severe asthma. Allergy Asthma Immunol Res. 2015;7(2):106-117. doi:10.4168/aair.2015.7.2.106

41. Lee HY, Lee GH, Kim HR, Lee YC, Chae HJ. Phosphatidylinositol 3-kinase-delta controls endoplasmic reticulum membrane fluidity and permeability in fungus-induced allergic inflammation in mice. Br J Pharmacol. 2020;177(7):1556-1567. doi:10.1111/bph.14917 
42. Di Conza G, Ho PC. ER stress responses: an emerging modulator for innate immunity. Cells. 2020;9:3. doi:10.3390/cells9030695

43. Klymenko O, Huehn M, Wilhelm J, et al. Regulation and role of the ER stress transcription factor CHOP in alveolar epithelial type-II cells. J Mol Med. 2019;97(7):973-990. doi:10.1007/s00109-01901787-9

44. Chen G, Ribeiro CMP, Sun L, et al. XBP1S regulates MUC5B in a promoter variant-dependent pathway in idiopathic pulmonary fibrosis airway epithelia. Am J Respir Crit Care Med. 2019;200 (2):220-234. doi:10.1164/rccm.201810-1972OC

45. Huang G, Su J, Zhao W, et al. JNK modulates RAGE/beta-catenin signaling and is essential for allergic airway inflammation in asthma. Toxicol Lett. 2021;336:57-67. doi:10.1016/j.toxlet.2020.10.002

46. Wu HM, Shen QY, Fang L, et al. JNK-TLR9 signal pathway mediates allergic airway inflammation through suppressing melatonin biosynthesis. J Pineal Res. 2016;60(4):415-423. doi:10.1111/ jpi.12323

47. Ma SQ, HL W, X Z. TLR2 regulates allergic airway inflammation through NF- $\mathrm{B}$ and MAPK signaling pathways in asthmatic mice. Eur Rev Med Pharmacol Sci. 2018;22(10):3138-3146.
48. Duan W, Chan JH, McKay K, et al. Inhaled p38alpha mitogen-activated protein kinase antisense oligonucleotide attenuates asthma in mice. Am J Respir Crit Care Med. 2005;171(6):571-578. doi:10.1164/rccm.200408-1006OC

49. Nath P, Leung SY, Williams A, et al. Importance of p38 mitogen-activated protein kinase pathway in allergic airway remodelling and bronchial hyperresponsiveness. Eur J Pharmacol. 2006;544 (1-3):160-167. doi:10.1016/j.ejphar.2006.06.031

50. Wang $\mathrm{N}$, Wang J, Zhang $\mathrm{Y}$, et al. Imperatorin ameliorates mast cell-mediated allergic airway inflammation by inhibiting MRGPRX2 and CamKII/ERK signaling pathway. Biochem Pharmacol. 2021;184:114401. doi:10.1016/j.bcp.2020.114401

51. Li Y, Jiang W, Niu Q, et al. eIF2alpha-CHOP-BCl-2/JNK and IRE1alpha-XBP1/JNK signaling promote apoptosis and inflammation and support the proliferation of Newcastle disease virus. Cell Death Dis. 2019;10(12):891. doi:10.1038/s41419-019-2128-6

\section{Publish your work in this journal}

The Journal of Asthma and Allergy is an international, peer-reviewed open-access journal publishing original research, reports, editorials and commentaries on the following topics: Asthma; Pulmonary physiology; Asthma related clinical health; Clinical immunology and the immunological basis of disease; Pharmacological interventions and new therapies. The manuscript management system is completely online and includes a very quick and fair peer-review system, which is all easy to use. Visit http://www.dovepress.com/testimonials.php to read real quotes from published authors. 